5. Hornick JL, Dal Cin P, Fletcher CD. Loss of INI1 expression is char acteristic of both conventional and proximal-type epithelioid sarcoma. Am J Surg Pathol 2009; 33: 542-50.

6. Antonescu CR, Zhang L, Chang NE, et al. EWSR1-POU5F1fusion in soft tissue myoepithelial tumors. A molecular analysis of sixty-six cases, including soft tissue, bone, and visceral lesions, showing common involvement of the EWSR1 gene. Genes Chromosomes Cancer 2010; 49: $1114-24$.

7. Okamoto S, Hisaoka M, Ishida T, et al. Extraskeletal myxoidchondrosarcoma: a clinicopathologic, immunohistochemical, and molecular analysis of 18 cases. Hum Pathol 2001; 32: 1116-24.

8. Oliveira AM, Sebo TJ, McGrory JE, et al. Extraskeletal myxoid chondrosarcoma: a clinicopathologic, immunohistochemical, and ploidy analysis of 23 cases. Mod Pathol 2000; 13: 900-8.

9. Fletcher CDM, Bridge JA, Hogendoorn PCW, et al. WHO Classification of Tumours of Soft Tissue and Bone. Lyon: IARC Press, 2013.

10. Flucke U, Hulsebos TJ, van Krieken JH, et al. Myxoid epithelial sarcoma: a diagnostic challenge. A report on six cases. Histopathology 2010; 57: 753-9.

DOI: https://doi.org/10.1016/j.pathol.2019.06.004

\section{Comparison of Vitek 2 YS08 with Sensititre YeastOne for Candida susceptibility testing}

Sir,

An early and accurate antifungal susceptibility result is important for the treatment of invasive candidiasis. Sensititre YeastOne (Thermo Scientific, USA) has good concordance with the standard Clinical and Laboratory Standards Institute (CLSI) reference method for Candida susceptibility testing, and therefore is a widely utilised commercial method of determining Candida susceptibility. The automated Vitek 2 AST YS08 (bioMérieux, France) has advantages in decreased turnaround time, reduced costs and ease of use; however, there are limited data regarding its performance for resistant isolates.

A total of 68 clinical isolates of Candida species, many known to have antifungal resistance, were tested by Vitek 2 AST YS08 and the Sensititre YeastOne method according to the manufacturer's instructions. They were comprised of Candida albicans $(n=20), \quad$ Candida glabrata $(n=21)$, Candida tropicalis $(n=9)$, Candida parapsilosis $(n=10)$ and Candida krusei $(n=8)$. Candida species were identified with a log score $>2.0$ using the MALDI Biotyper (Bruker, USA).

Essential agreement was defined as $\leq 2$ minimum inhibitory concentration (MIC) dilution difference and categorical agreement was obtained when the MIC result fell within the same interpretive categories according to CLSI breakpoints for azoles and echinocandins, ${ }^{2}$ and according to the epidemiological cutoff values for amphotericin $\mathrm{B}^{3}$ Very major errors, major errors and minor errors were defined according to a prior study, with Sensititre YeastOne as the reference method. ${ }^{4}$ Very major errors occurred where the reference method categorised the isolate as resistant and Vitek 2 categorised it as susceptible. Major errors occurred where the reference method categorised the isolate as susceptible and Vitek 2 categorised it as resistant. Minor errors occurred where one of the methods categorised the isolate as susceptible or resistant and the other method categorised the isolate as intermediate or susceptible dose dependent.
Table 1 demonstrates that essential agreement and categorical agreement were suboptimal for fluconazole and voriconazole with some very major errors, while there was good agreement for the other antifungals tested, acknowledging the lack of isolates which were non-susceptible to micafungin and amphotericin. Poor agreement for fluconazole was largely found in $C$. albicans where essential agreement occurred in 14/20 isolates and categorical agreement in 17/20 isolates. Poor agreement for voriconazole was predominantly found in $C$. krusei where essential agreement occurred in $2 / 8$ isolates and categorical agreement occurred in only $2 / 8$ isolates, mostly classified as minor errors. Caspofungin non-susceptibility occurred predominantly in C. glabrata complex and it was in this species complex that poor categorical agreement for caspofungin occurred with essential agreement in 15/15 isolates and categorical agreement in 5/15 isolates, mostly classified as minor errors.

Many previous studies of Vitek 2 Candida susceptibility testing had a low percentage of resistant isolates, ${ }^{5-7}$ hampering the comparison to our study; however, some studies which were enriched for resistant isolates like ours have shown better performance. Cuenca-Estrella et al. tested a set of 154 Candida isolates, approximately half with elevated azole MICs. ${ }^{8}$ They found essential agreement between Vitek 2 and the formal CLSI method of $>96 \%$ for fluconazole and voriconazole. Using old CLSI breakpoints for fluconazole, minor errors occurred for 25 isolates, and major or very major errors in three isolates. For voriconazole, minor errors occurred for two isolates, and major or very major errors for four isolates. Posteraro et al. found good essential agreement and categorical agreement for their set which included 11 azole resistant $C$. albicans and 48 azole resistant $C$. glabrata. ${ }^{4}$ The better essential agreement seen in these two studies compared to our study may have been influenced by the Candida species which were tested. Both studies included an assessment of fluconazole and

Table 1 Comparison of Vitek 2 AST YS08 to reference method Sensititre YeastOne Candida susceptibility testing

\begin{tabular}{|c|c|c|c|c|c|c|c|c|}
\hline \multirow[t]{2}{*}{$\begin{array}{l}\text { Antifungal } \\
\text { agents }\end{array}$} & \multirow[t]{2}{*}{$\begin{array}{l}\text { Isolates }^{\mathrm{a}} \\
(n)\end{array}$} & \multirow[t]{2}{*}{$\begin{array}{c}\text { Resistant }^{\mathrm{b}} \\
(n)\end{array}$} & \multirow[t]{2}{*}{$\begin{array}{l}\mathrm{I} / \mathrm{SDD}^{\mathrm{b}} \\
(n)\end{array}$} & \multirow[t]{2}{*}{$\begin{array}{l}\text { EA } \\
(\%)\end{array}$} & \multirow[t]{2}{*}{$\begin{array}{l}\text { CA } \\
(\%)\end{array}$} & \multicolumn{3}{|c|}{$\begin{array}{l}\text { Misclassified } \\
\text { isolates }(n)\end{array}$} \\
\hline & & & & & & VME & $\mathrm{ME}$ & $\mathrm{mE}$ \\
\hline Fluconazole & 39 & 18 & 1 & 77 & 90 & 2 & 0 & 2 \\
\hline Voriconazole & 47 & 14 & 11 & 79 & 66 & 2 & 1 & 13 \\
\hline Caspofungin & 62 & 3 & 7 & 100 & 82 & 0 & 2 & 9 \\
\hline Micafungin & 54 & 1 & 0 & 100 & 100 & 0 & 0 & 0 \\
\hline Amphotericin & 67 & 0 & 0 & 100 & 100 & 0 & 0 & 0 \\
\hline Flucytosine ${ }^{c}$ & 68 & NA & NA & 99 & NA & NA & $\mathrm{NA}$ & NA \\
\hline
\end{tabular}

$\mathrm{CA}$, categorical agreement; EA, essential agreement; $\mathrm{mE}$, minor errors; $\mathrm{ME}$ major errors; VME, very major errors.

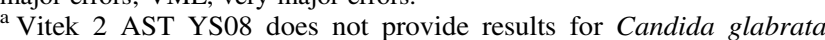
against fluconazole and voriconazole, nor for Candida krusei agains fluconazole. Six isolates for caspofungin and one isolate for amphotericin terminated by Vitek AST YS08 so did not allow comparison. Fourteen isolates were not tested for micafungin by YeastOne so did not allow comparison.

${ }^{\mathrm{b}}$ Resistant or intermediate/susceptible dose dependent according to Sensititre YeastOne results with CLSI interpretative criteria.

${ }^{\mathrm{c}}$ There is no CLSI breakpoint or epidemiological cutoff value for flucystosine, therefore no CA was calculated. 
voriconazole for $C$. glabrata complex, which we could not assess as the current Vitek 2 system issues no result for fluconazole or voriconazole for this species complex. Additionally, Posteraro et al. did not test C. krusei which in our study had poor essential agreement for voriconazole. Categorical agreement between Vitek 2 and Sensititre YeastOne for caspofungin was noted to be suboptimal in our study, congruent with Astvad et al. who found 6/31 fks mutant Candida isolates were misclassified as susceptible by the Vitek 2 system, while the reference CLSI and European Committee on Antimicrobial Susceptibility Testing (EUCAST) methods misclassified $<4 \%$ of these isolates.

In our study skewed for resistant Candida isolates, essential agreement and categorical agreement between Vitek 2 AST YS08 and Sensititre YeastOne were suboptimal. More validation data with resistant isolates needs to be obtained for the Vitek 2 AST YS08 system.

Conflicts of interest and sources of funding: BioMerieux supplied Vitek 2 AST YS08 cards free of charge. The authors state that there are no conflicts of interest to disclose.

\section{Ka Yan Wong, Dianne Gardam, Peter Boan}

Department of Microbiology, PathWest Laboratory Medicine WA, Fiona Stanley Hospital, Murdoch, WA, Australia

Contact Peter Boan.

E-mail: Peter.Boan@health.wa.gov.au

1. Posteraro B, Spanu T, Fiori B, et al. Antifungal susceptibility profiles of bloodstream yeast isolates by Sensititre YeastOne over nine years at a large Italian teaching hospital. Antimicrob Agents Chemother 2015; 59: 3944-55.

2. Clinical and Laboratory Standards Institute (CLSI). M60. Performance standards for antifungal susceptibility testing of yeasts. 1st ed. Wayne, PA: CLSI, 2017.

3. Clinical and Laboratory Standards Institute (CLSI). M59. Epidemiological cutoff values for antifungal susceptibility testing. 2nd ed. Wayne, PA CLSI, 2018.

4. Posteraro B, Martucci R, La Sorda M, et al. Reliability of the Vitek 2 yeast susceptibility test for detection of in vitro resistance to fluconazole and voriconazole in clinical isolates of Candida albicans and Candida glabrata. J Clin Microbiol 2009; 47: 1927-30.

5. Pfaller MA, Diekema DJ, Procop GW, et al. Multicenter comparison of the VITEK 2 antifungal susceptibility test with the CLSI broth microdilution reference method for testing amphotericin B, flucytosine, and voriconazole against Candida spp. J Clin Microbiol 2007; 45: 3522-8.

6. Pfaller MA, Diekema DJ, Procop GW, et al. Multicenter comparison of the VITEK 2 yeast susceptibility test with the CLSI broth microdilution reference method for testing fluconazole against Candida spp. J Clin Microbiol 2007; 45: 796-802.

7. Peterson JF, Pfaller MA, Diekema DJ, et al. Multicenter comparison of the Vitek 2 antifungal susceptibility test with the CLSI broth microdilution reference method for testing caspofungin, micafungin, and posaconazole against Candida spp. J Clin Microbiol 2011; 49: 1765-71.

8. Cuenca-Estrella M, Gomez-Lopez A, Alastruey-Izquierdo A, et al. Comparison of the Vitek 2 antifungal susceptibility system with the clinical and laboratory standards institute (CLSI) and European Committee on Antimicrobial Susceptibility Testing (EUCAST) Broth Microdilution Reference Methods and with the Sensititre YeastOne and Etest techniques for in vitro detection of antifungal resistance in yeast isolates. J Clin Microbiol 2010; 48: 1782-6.

9. Astvad KM, Perlin DS, Johansen HK, et al. Evaluation of caspofungin susceptibility testing by the new Vitek 2 AST-YS06 yeast card using a unique collection of FKS wild-type and hot spot mutant isolates, including the five most common candida species. Antimicrob Agents Chemother 2013; 57: 177-82.

DOI: https://doi.org/10.1016/j.pathol.2019.05.005

\section{A descriptive account of sequential nailfold capillaroscopy in scleroderma}

Sir,

Microvasculopathy is an early and prominent pathological feature of systemic sclerosis ( $\mathrm{SSc}$ ), or scleroderma, and is most easily recognised in the capillaries of the nailfold using the simple technique of nailfold capillaroscopy (NFC). ${ }^{1}$ Many studies have confirmed that NFC has proven utility in the early diagnosis of SSc and it has been included in the classification criteria for this disease since 1988. ${ }^{1}$ However, to date there is little information concerning sequential NFC in scleroderma and whether documentation of nailfold capillary morphology and density over time may assist in disease management and prognosis.

In this current study we have compared and contrasted sequential nailfold capillary density and morphological characteristics in both healthy subjects and scleroderma patients for periods of up to 12 months.

There were nine patients in the scleroderma study group, three with diffuse cutaneous scleroderma, five with limited cutaneous scleroderma and one with overlap scleroderma. All patients were recruited from the South Australian Scleroderma Register and all patients fulfilled the diagnosis of scleroderma according to the American College of Rheumatology/European League Against Rheumatism (ACR/EULAR) 2013 classification criteria and stratified as limited cutaneous, diffuse cutaneous or overlap scleroderma according to LeRoy's criteria. ${ }^{2-4}$ The demographics, clinical and serological features and medications of the scleroderma study group (at entry to the study) are shown in Table 1.

There were four healthy control subjects, three females and one male with an age span of 28-66 years. None were smokers and none had Raynaud's phenomenon. NFC was performed on both patients and controls at regular intervals over the year study period.

NFC was performed on the fourth finger nailfold of each hand using a Capiscope (supplied by KK Technology, United Kingdom). Paraffin oil or KY jelly was applied to the nailbed to reduce the skin/air refractive barrier. The Capiscope technique allows visualisation of the nailfold capillaries at magnifications of $100 \times$ and $300 \times$ and has the capability of digitalisation of the nailfold images or video capillaroscopy. The procedure was done exactly as described in the Capiscope user's manual enabling the capture of multiple digitalised images. ${ }^{5}$ Overlapping images were then aligned and electronically spliced to form a composite mosaic of the nailfold and its capillary arcades. The microvasculature was then assessed from the digitalised images. In particular, we assessed either quantitatively or qualitatively the symmetry or otherwise of the capillary arcades, the morphology and dimensions [normal size dilated or grossly dilated (giant) of the capillary loops, nailfold capillary density, the presence and appearances of capillary microbleeds and the appearance of the cuticle (widened, roughened, discoloured)].

Capillary density was measured from the digitalised nailfold images of each nailfold by the direct observation method 